\title{
Personality and Temperament Correlates of Pain Catastrophizing in Young Adolescents
}

\author{
Peter Muris · Cor Meesters · Anja van den Hout • Sylvia Wessels • \\ Ingmar Franken · Eric Rassin
}

Received: 12 July 2006/Accepted: 12 March 2007/Published online: 4 April 2007

(C) Springer Science+Business Media, LLC 2007

\begin{abstract}
Pain catastrophizing is generally viewed as an important cognitive factor underlying chronic pain. The present study examined personality and temperament correlates of pain catastrophizing in a sample of young adolescents $(N=132)$. Participants completed the Pain Catastrophizing Scale for Children, as well as scales for measuring sensitivity of the behavioral inhibition and behavioral activation systems (BIS-BAS), and various reactive and regulative temperament traits. Results demonstrated that BIS, reactive temperament traits (fear and anger-frustration), and perceptual sensitivity were positively related to pain catastrophizing, whereas regulative traits (attention control, inhibitory control) were negatively associated with this cognitive factor. Further, regression analyses demonstrated that only BIS and the temperamental traits of fear and perceptual sensitivity accounted for a unique proportion of the variance in adolescents' pain catastrophizing scores.
\end{abstract}

Keywords Temperament $\cdot$ Personality · Pain catastrophizing · Adolescents

\section{Introduction}

Research has shown that a substantial minority of the non-referred children and adolescents $(25 \%)$ report chronic levels of pain [1-3]. Theories of chronic pain propose that pain is a complex phenomenon that is affected by biological, psychological and social processes

P. Muris $(\bowtie) \cdot$ I. Franken · E. Rassin

Institute of Psychology, Erasmus University Rotterdam, P.O. Box 1738, 3000 DR Rotterdam,

The Netherlands

e-mail: muris@fsw.eur.nl

C. Meesters - A. van den Hout $\cdot$ S. Wessels

Department of Medical, Clinical and Experimental Psychology, Maastricht University,

Maastricht, The Netherlands 
[4-7]. This biopsychosocial perspective is echoed in current cognitive-behavioral models of chronic pain, which have implicated fear of pain as an important vehicle behind the development and maintenance of chronic pain [8,9].

One important cognitive factor that is thought to fuel fear of pain is pain catastrophizing [9], which can be defined as the tendency to focus on pain and negatively evaluate one's ability to deal with pain. There is abundant evidence that catastrophizing is significantly associated with pain in adults [10]. However, few studies have examined pain catastrophizing in children. One exception is a study by Crombez et al. [11] who demonstrated that this construct can be reliably assessed in youths from age eight on. Furthermore, these researchers showed that catastrophizing made unique contributions to pain intensity and disability. Another study by Eccleston et al. [12] showed that catastrophizing was one of the most powerful predictors of symptoms of emotional distress (i.e., anxiety and depression) in a sample of clinically referred adolescents suffering from chronic pain.

It is generally assumed that neuroticism is involved in the exacerbation of pain. Briefly, neuroticism can be defined as the trait-like tendency to experience a broad range of negative feelings such as anxiety and distress [13]. As neuroticism is significantly associated with worry [14], it is not surprising that this personality factor is thought to be linked to pain catastrophizing. Direct evidence for this idea comes from a study by Goubert et al. [15] who assessed neuroticism, pain catastrophizing, and vigilance to pain in a sample of adult chronic low back pain patients. Structural equations modeling yielded support for a model in which neuroticism was indeed associated with pain catastrophizing, which in turn was linked to heightened levels of vigilance to pain.

While it has been demonstrated that neuroticism is also a significant predictor of chronic pain complaints in children and adolescents [16], so far no study has examined the relation between this personality variable and pain catastrophizing in young people. The present study was set up to investigate this issue. Non-referred adolescents completed a child measure of pain catastrophizing as well as questionnaires measuring important aspects of neuroticism. Two temperament/personality theories, in which neuroticism-like traits play a key role, had our specific attention. The first one is the biological personality theory of Gray [17, 18], which posits two main brain systems that regulate behaviour in response to environmental stimuli. The behavioural inhibition system (BIS) serves to alert the person to the possibility of danger or punishment, thereby enhancing avoidance behaviour, whereas the behavioural activation system (BAS) is sensitive to signals of reward, and involved in approach behaviour. As BIS shows strong similarity to neuroticism [19, 20], the present study hypothesized that this personality factor would be positively related to pain catastrophizing.

The second theory is Rothbart's temperament model [21]. According to this model, temperament can be defined as individual differences in reactivity and self-regulation. Reactivity primarily refers to negative affectivity, which can be regarded as an equivalent of neuroticism, and consists of two important lower-order traits, namely fear and angerfrustration, of which the former seems most relevant in the case of an anxiety-related phenomenon such as pain catastrophizing. Self-regulation refers to effortful control, which pertains to executive functioning-based processes that regulate children's behavior and thoughts, and as such low levels of this temperament factor have been associated with high levels of uncontrollable dysfunctional thoughts [22]. Thus, it can be expected that negative affectivity and in particular the lower-order component of fear would be positively linked to pain catastrophizing, while effortful control variables would be negatively associated with this cognitive factor. 
Besides reactive and regulative temperament traits referring to respectively negative affectivity and effortful control, Rothbart's model contains various other temperamental traits of which most are not particularly relevant when studying youths' responses to pain experiences [21]. An exception might be a trait named 'perceptual sensitivity', which can be defined as "the detection or perceptual awareness of slight, low-intensity stimulation". Obviously, it may well be the case that children and adolescents who score high on this trait are more prone to experience pain, and possibly are more susceptible to processes like pain catastrophizing [23]. In other words, the temperamental trait of perceptual sensitivity was anticipated to be positively related to pain catastrophizing.

To summarize, pain catastrophizing is thought to play an important role in the development of chronic pain. More precisely, it is assumed that this cognitive factor enhances vigilance and fear of pain, which in turn may result in avoidance behaviour and ultimately chronic pain complaints. As shown in Fig. 1, the present study examined various temperament/personality factors that are possibly associated with pain catastrophizing, namely neuroticism-like traits such as BIS and fear, low effortful control, and perceptual sensitivity.

\section{Method}

Participants and procedure

Two-hundred-and-fifty first-year students of a regular secondary school in the southern part of the Netherlands were invited to participate in this study. This was done by sending them and their parents an informed consent letter. One-hundred-and-thirty-two of them (71 boys and 61 girls; $52.8 \%$ ) decided to participate and completed the set of questionnaires during regular classes. Adolescents attended either higher general secondary education or preuniversity education, indicating above-average to high intellectual functioning. All participants were either 12 or 13 years old (mean age $=12.55$ years, $\mathrm{SD}=0.52$ ). From middle childhood on, children's cognitive abilities show a significant increase, which enhance their proneness to engage in negative thought processes [24], and thus it can be expected that the young adolescents in the present study possibly display catastrophizing responses to pain. No exact information about the socioeconomic status of the adolescents was available. Based on the educational levels of both parents, percentages of participants with

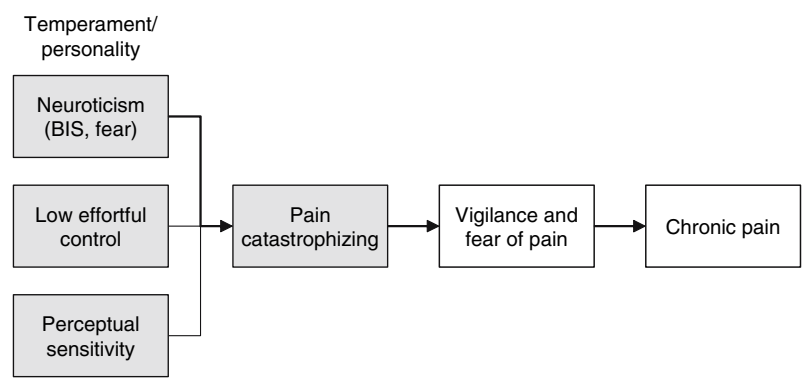

Fig. 1 Hypothesized model for the relationships between various temperament/personality factors, pain catastrophizing, fear of pain, and chronic pain. The grey rectangles in the model are investigated in the present study 
a low, middle, or high socioeconomic background were estimated 20, 35, and 45\%, respectively. The vast majority of the youths was Caucasian $(>90 \%)$. The study was reviewed and approved by the ethic board for psychological research of Erasmus University.

\section{Questionnaires}

Pain catastrophizing was measured with the child version of the Pain Catastrophizing Scale (PCS-C [11, 25]). The scale consists of 13 items (e.g., "When I feel pain, I keep thinking about how much it hurts') that have to be answered on a 5-point scale with $0=$ not at all, $1=$ mildly, $2=$ moderately, $3=$ severely, and $4=$ extremely). Scores are summed to yield a total score (range: 0-52), with higher scores indicating higher levels of pain catastrophizing. Research has demonstrated that the PCS-C is a reliable instrument with satisfactory predictive validity [11, 26, 27].

The child version of the BIS/BAS scales $[28,29]$ intends to assess individual differences in sensitivity of the BIS and BAS. The 20 items of the original scales were somewhat changed and simplified to make them more appropriate and understandable to children (e.g., "I am hurt when people scold me or tell me that I do something wrong", "I get thrilled when good things happen to me" [29]). Each item has to be scored on a four-point Likert scale with $0=$ not true, $1=$ somewhat true, $2=$ true, and $3=$ very true. Total BIS and BAS scores can be obtained by summing across relevant items. It has been shown that the child version of the BIS/BAS scales possesses a theoretically meaningful factor structure with two separate factors for BIS and BAS. Further, the reliability of the BIS and BAS scales appears satisfactory, and the scales generally show the expected relationships with Eysenck's personality traits of neuroticism and extraversion and psychopathological symptoms [29].

The Revised version of the Early Adolescent Temperament Questionnaire (EATQ-R [30, 31] contains 103 items and measures a wide range of children's temperamental characteristics. The present study examined the role of temperamental aspects that may be relevant to the experience of pain. More precisely, the study focused on the negative affectivity traits of 'fear' (six items; i.e., the tendency to experience unpleasant affect related to anticipation of distress) and 'anger-frustration' ( 9 items; i.e., the tendency to experience unpleasant affect related to the interruption of ongoing tasks or goal blocking), the effortful control traits of 'activation control' (eight items; i.e., the ability to perform an action when there is a strong tendency to avoid it), 'attention control' (seven items; i.e., the ability to focus and shift attention when desired), and 'inhibitory control' (11 items; i.e., the ability to plan, and to suppress inappropriate responses), as well as the temperament trait of 'perceptual sensitivity' (six items; i.e., the detection and awareness of slight, lowintensity sensory stimulation), which might be especially relevant to the perception of pain. Items have to be answered on a 5-point Likert scale ranging from $1=$ almost never true to $5=$ almost always true. For each trait, a total score can be computed by summing ratings across relevant items (after recoding inversely formulated items). Previous research has provided tentative evidence for the psychometric properties of the EATQ-R [31]. That is, the internal consistency of the instrument appeared sufficient (with Cronbach's alphas varying between 0.65 and 0.82 ), whereas the inter-rater agreement was modest (with parent-child correlations in the $0.20-0.40$ range).

The Strengths and Difficulties Questionnaire (SDQ [32]) consists of 25 items describing positive and negative attributes of children and adolescents that can be allocated to five 
subscales of five items each: emotional symptoms, conduct problems, hyperactivity-inattention, peer problems, and prosocial behavior. Each item has to be scored on a 3-point scale with $0=$ not true, $1=$ somewhat true, and $2=$ certainly true. Research by Goodman and colleagues has shown that the SDQ possesses good psychometric properties [32]. In the present study, the emotional symptoms scale (range 0-10) was used. As research has demonstrated that pain and in its wake pain catastrophizing are substantially related to symptoms of anxiety and depression [33,34], it seemed appropriate to investigate to what extent temperament and personality variables contribute to pain catastrophizing beyond the influence of such symptoms.

\section{Results}

\section{General findings}

Before presenting the main results of the current study, a number of general findings should be discussed. First, most questionnaires that were used in this investigation were found to possess satisfactory internal consistency (see Table 1). Reliability coefficients of the EATQ scales were sufficient but modest (all alphas were between 0.60 and 0.68 ), although it should be borne in mind that these scales only consisted of a limited set of items. Second, $t$ tests indicated that there was only one significant gender difference: That is, boys displayed higher BAS scores as compared to girls, means being $20.56(\mathrm{SD}=5.63)$ versus 18.11 $(\mathrm{SD}=4.78)[t(130)=2.67, P<0.01]$. Third, a comparison with normative data of the PCS$\mathrm{C}$ as provided by Crombez et al. [11] revealed that the adolescents in the present sample displayed relatively low pain catastrophizing scores (means being 9.14 in the present sample versus 16.76 in the sample of Crombez et al.).

Personality, temperament, and children's pain catastrophizing

As expected, BIS was significantly related to children's pain catastrophizing scores $(r=0.56, P<0.001)$. However, BAS was also positively linked to PCS-C scores $(r=0.27$, $P<0.05)$. Correlations between EATQ-R temperament scales and PCS-C generally showed the predicted pattern. That is, the negative affectivity traits of fear and angerfrustration were positively related to children's pain catastrophizing scores ( $r$ s being 0.49 and 0.44 , respectively, both $P \mathrm{~s}<0.001$ ), whereas the regulative traits of attention control and inhibitory control were negatively associated with such scores (both $r$ s being -0.23 , $P$ s $<0.05)$. Furthermore, a small but significant positive correlation was found between perceptual sensitivity and children's pain catastrophizing scores $(r=0.26, P<0.05)$. When adopting a Bonferroni procedure (in order to correct for the large number of correlations that were computed), in particular neuroticism-based variables such as BIS and EATQ negative affectivity traits remained significantly linked to PCS-C scores (see Table 1).

To examine the unique contributions of various personality and temperament factors to children's pain catastrophizing scores, a hierarchical regression analysis was carried out. In this analysis, age and gender were forced into the equation on step 1 (as previous research with the PCS-C has demonstrated that girls and younger youths display somewhat higher pain catastrophizing scores as compared to boys and older youths [11]), whereas all personality and temperament variables were entered on step 2. Collinearity statistics were 


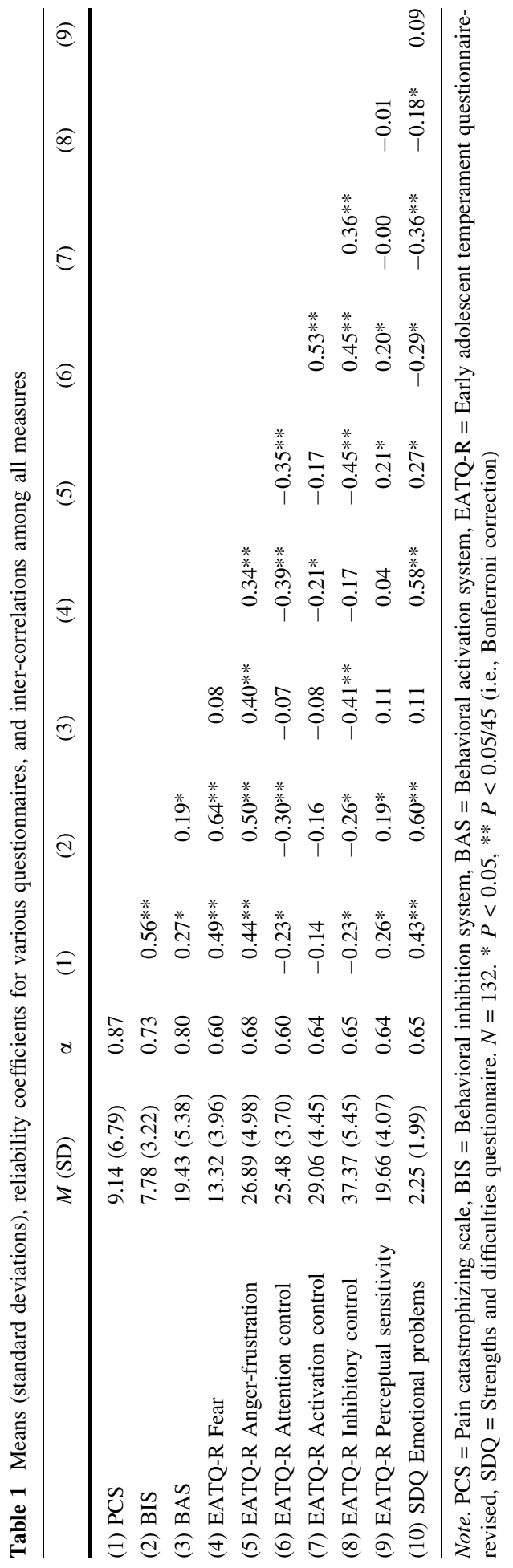


Table 2 Results of the hierarchical regression analysis predicting children's PCS pain catastrophizing scores from personality and temperament factors

\begin{tabular}{llrrrc}
\hline Step & Predictor & \multicolumn{1}{l}{$B$} & \multicolumn{1}{l}{ SE } & \multicolumn{1}{l}{$\beta$} & $\Delta R^{2}$ \\
\hline 1 & Age & -1.75 & 1.15 & -0.13 & 0.02 \\
& Gender & -0.84 & 1.18 & -0.06 & \\
& BIS & 0.57 & 0.21 & $0.27^{* * *}$ & $0.40^{* * * *}$ \\
& BAS & 0.18 & 0.10 & 0.14 & \\
& EATQ-R Fear & 0.41 & 0.16 & $0.24^{*}$ & \\
& EATQ-R Anger-frustration & 0.18 & 0.13 & 0.13 & \\
& EATQ-R Attention control & -0.10 & 0.18 & -0.06 & \\
& EATQ-R Activation control & 0.00 & 0.13 & 0.02 & \\
& EATQ-R Inhibitory control & 0.00 & 0.11 & 0.03 & \\
& EATQ-R Perceptual sensitivity & 0.27 & 0.13 & $0.16^{*}$ & \\
&
\end{tabular}

Note. PCS $=$ Pain catastrophizing scale, BIS $=$ Behavioral inhibition system, $\mathrm{BAS}=$ Behavioral activation system, EATQ-R $=$ Early adolescent temperament questionnaire-revised. $N=132$. $* P<0.05$, $* * P<0.01$, $* * * P<0.001$

inspected and were all found to be within acceptable limits (i.e., for all predictor variables, tolerance (TOL) was $>0.50$, whereas the variance of inflation factor (VIF) was $<2.50$ ). As can be seen in Table 2 , results indicated that BIS $(\beta=0.27)$, EATQ-R fear $(\beta=0.24)$, and EATQ-R perceptual sensitivity $(\beta=0.16)$ each made a unique and significant contribution to children's pain catastrophizing scores. Together, personality and temperament traits accounted for $40 \%$ of the total variance in PCS-C scores.

As expected, pain catastrophizing was significantly related to SDQ emotional symptoms $(r=0.43, P<0.001)$. An additional regression analysis was conducted to investigate to what extent temperament and personality variables contributed to pain catastrophizing beyond the influence of such symptoms. Results indicated that when controlling for age and gender (step 1) and SDQ emotional symptoms (step 2), BIS, EATQ-R fear, and EATQ-R perceptual sensitivity (step 3) still made significant contributions to children's PCS-C scores (the $\beta$ s were $0.23,0.21$, and 0.16 , respectively).

\section{Discussion}

The current study examined personality and temperament correlates of pain catastrophizing in a sample of young adolescents. The results can be catalogued as follows. First, PCS-C scores were related to various personality and temperament variables in a theoretically meaningful way. That is, positive correlations were found between pain catastrophizing and BIS, the reactive traits of fear and anger-frustration, and perceptual sensitivity, whereas negative correlations emerged between pain catastrophizing and the regulative traits of attention control and inhibitory control. Second, regression analyses demonstrated that only BIS and the temperamental traits of fear and perceptual sensitivity accounted for a unique proportion of the variance in adolescents' pain catastrophizing scores, and these contributions remained significant even when controlling for concurrent emotional symptoms.

Previous studies in adult and child populations have shown that neuroticism increases vulnerability to chronic pain [16, 35]. Furthermore, Goubert et al. [15] found evidence in 
adults that this link is mediated by the cognitive factor of pain catastrophizing. The present study showed that, in adolescents, in particular the personality factor of BIS and the temperamental trait of fear are associated with high levels of pain catastrophizing. Clearly, BIS and fear seem to represent the vulnerability factor of neuroticism, and as such these findings are well in keeping with those reported by Goubert et al. [15]. The question remains why neuroticism is related with high levels of pain catastrophizing. This has probably to do with neurotic people's tendency to evaluate threatening stimuli as more probable, imminent, and aversive, and to perceive coping possibilities as less effective, which according to Tallis and Eysenck [36] would result in increased worry and eventually catastrophizing.

Although effortful control-based temperament characteristics were negatively correlated with adolescents' pain catastrophizing scores, this link disappeared when controlling for neuroticism-related traits in the regression analysis. This finding is in contrast with recent studies showing that reactive (neuroticism) and regulative (effortful control) temperament traits each make a unique contribution to symptoms of anxiety, depression, and aggression [22]. It may well be the case that the effects of regulative temperament factors on pain catastrophizing only become evident when individuals are actually confronted with pain. There is recent evidence indicating that this idea might be correct. In a sample of patients with rheumatoid arthritis, Hamilton et al. [37] demonstrated that while neuroticism had a negative impact on mood, regulative personality characteristics indeed promoted adaptation to pain exacerbations. This finding at least demonstrates that not only reactive but also regulative traits are relevant for individuals' responses to pain, but the effects of both types of traits on pain catastrophizing clearly need further study.

An unexpected finding of the present study was that BAS was positively associated with pain catastrophizing scores. This result was probably caused by the fact that there was a small but significant positive correlation between BIS and BAS. It seems most plausible that this result reflects children's susceptibility to response tendency, that is the inclination to score consistently lower or higher on the items of one and the same questionnaire [29]. In keeping with this idea, the present data showed that when controlling for the shared variance among BIS and BAS, only BIS remained significantly related to PCS-C scores, whereas BAS was no longer linked to pain catastrophizing.

The temperamental trait of perceptual sensitivity had a unique influence on adolescents' level of pain catastrophizing. This finding seems to make sense: perceptual sensitivity refers to the detection and awareness of low-intensity sensory stimuli, and these may include mild pain. Increased perception of pain symptoms may be associated with increased catastrophizing and fear of such symptoms, in particular in subjects who are characterized by high levels of neuroticism $[23,38]$. Perceptual sensitivity in relation to pain comes close to what has been labelled as pain vigilance in the adult literature. Various studies have shown that pain vigilance, which is intimately related to pain catastrophizing, plays a key role in the aetiology of chronic pain [39, 40].

It should be acknowledged that the present investigation suffered from various limitations. As mentioned earlier, no measure was included for measuring adolescents' actual frequency and/or severity of pain. Further, the study was cross-sectional in nature and therefore it is not possible to make causal attributions about the link between personality and temperament characteristics and pain catastrophizing. Further prospective research is urgently needed to demonstrate the precise role of pain catastrophizing in the hypothesized model of chronic pain (see Fig. 1), and to exclude the possibility that this cognitive variable is merely an epiphenomenon in youths who already suffer from serious pain complaints. In addition, only half of the adolescents who were approached for the study 
eventually agreed to participate. Further, it should be borne in mind that the participants were non-referred (with fairly low pain catastrophizing scores), predominantly Caucasian, and intellectually high functioning. In other words, it remains to be seen whether the present findings can be replicated in clinically referred children and adolescents, and/or youths with a non-Caucasian ethnic background and lower intellectual abilities. Finally, only self-report scales were used for measuring adolescents' personality, temperament, and pain catastrophizing. Cross-validational information from the adolescents' parents, teachers, peers, and/or physicians would certainly have strengthened the notion that there is a meaningful link between temperament and personality traits and pain catastrophizing.

\section{Summary}

Taken together, the current study showed that neuroticism-related personality and temperament traits account for a substantial proportion of the variance in pain catastrophizing, which is generally considered as an important cognitive factor underlying chronic pain [41]. As to the practical implications of these findings, it should be borne in mind that neuroticism is a distal factor that is considered to enhance vulnerability to develop catastrophic thoughts about pain. Catastrophizing should be viewed as a proximal variable that is directly related to pain, disability, and distress. As such, it seems more appropriate for clinicians to screen for pain catastrophizing, as this factor provides a more direct indication of chronic pain complaints. Moreover, pain catastrophizing seems to be a clear target for cognitive-behavioral interventions, which have proven to be highly effective in treating chronic pain in youths [42]. On the other hand, for prevention workers, it may be useful to focus on temperament and personality factors, as they are probably more interested in the early detection of youths that might be prone to develop into pain catastrophizers.

\section{References}

1. Perquin CW, Hazebroek-Kampschreur AA, Hunfeld JA, Bohnen AM, Van Suijlekom-Smit LW, Passchier J, Van der Wouden JC (2000) Pain in children and adolescents: a common experience. Pain 87:51-58

2. Perquin CW, Hunfeld JA, Hazebroek-Kampschreur AA, Van Suijlekom-Smit LW, Passchier J, Koes BW, Van der Wouden JC (2001) Insights in the use of health care services in chronic benign pain in childhood and adolescence. Pain 94:205-213

3. Perquin CW, Hunfeld JA, Hazebroek-Kampschreur AA, Van Suijlekom-Smit LW, Passchier J, Koes BW, Van der Wouden JC (2003) The natural course of chronic benign pain in childhood and adolescence: a two-year population-based follow-up study. Eur J Pain 7:551-559

4. Asmundson G, Wright KD (2004) Biopsychosocial approaches to pain. In: Hadjistavropoulos T, Craig KD (eds) Pain: psychological perspectives. Lawrence Erlbaum, Mahwah, NJ, pp 35-57

5. Gatchel RJ (2004) The biopsychosocial perspective of pain. In: Raczynski JM, Leviton LC (eds) Handbook of clinical health psychology. American Psychological Association, Washington, pp 357-378

6. Keefe F, France C (1999) Pain: biopsychosocial mechanisms and management. Curr Dir Psychol Sci 8:137-141

7. Turk DC, Monarch ES (2002) Biopsychosocial perspective on chronic pain. In: Turk DC, Gatchel RJ (eds) Psychological approaches to pain management: a practitioner's handbook. Guilford Press, New York, pp 3-29

8. Asmundson G, Norton P, Norton G (1999) Beyond pain: the role of fear and avoidance in chronicity. Clin Psychol Rev 19:97-119

9. Vlaeyen J, Linton S (2000) Fear-avoidance and its consequences in chronic musculoskeletal pain: a state of the art. Pain 85:317-332 
10. Sullivan M, Thorn B, Haythornthwaite J, Keefe F, Martin M, Bradley L, Lefebvre J (2001) Theoretical perspectives on the relation between catastrophizing and pain. Clin J Pain 17:52-64

11. Crombez G, Bijttebier P, Eccleston C, Mascagni T, Mertens G, Goubert L, Verstraeten K (2003) The child version of the pain catastrophizing scale (PCS-C): a preliminary validation. Pain 104:639-646

12. Eccleston C, Crombez G, Scotford A, Clinch J, Connell H (2004) Adolescent chronic pain: patterns and predictors of emotional distress in adolescents with chronic pain and their parents. Pain 108:221-229

13. Eysenck HJ (1990) Genetic and environmental contributions to individual differences: the three major dimensions of personality. J Pers 58:245-261

14. Eysenck MW (1992) Anxiety. The cognitive perspective. Lawrence Erlbaum, Hove, UK

15. Goubert L, Crombez G, Van Damme S (2004) The role of neuroticism, pain catastrophizing and painrelated fear in vigilance to pain: a structural equations approach. Pain 107:234-241

16. Merlijn VP, Hunfeld JA, Van der Wouden JC, Hazebroek-Kampschreur AA, Koes BW, Passchier J (2003) Psychosocial factors associated with chronic pain in adolescents. Pain 101:33-43

17. Gray JA (1987) The psychology of fear and stress. Cambridge University Press, New York

18. Gray JA (1991) The neuropsychology of temperament. In: Strelau J, Angleiter A (eds) Explorations in temperament: international perspectives in theory and measurement. Plenum, New York, pp 105-128

19. Caseras X, Avila C, Torrubia R (2003) The measurement of individual differences in behavioural inhibition and behavioural activation systems: a comparison of personality scales. Pers Individ Diff 34:999-1013

20. Franken IHA, Muris P, Rassin E (2005) Psychometric properties of the Dutch BIS/BAS Scales. J Psychopath Behav Assess 27:25-30

21. Rothbart MK, Bates JE (1998) Temperament. In: Eisenberg N, Damon W (eds) Handbook of child psychology: social, emotional, and personality development, vol 3. Wiley, New York, pp 105-176

22. Muris P, Ollendick TH (2005) The role of temperament in the etiology of child psychopathology. Clin Child Fam Psychol Rev 8:271-289

23. Miller SM, Roussi P, Caputo C, Kruus L (1995) Pattern of children's coping with an aversive dental treatment. Health Psychol 14:236-246

24. Muris P, Merckelbach H, Meesters C, Van den Brand K (2002) Cognitive development and worry in normal children. Cogn Ther Res 26:775-785

25. Sullivan M, Bishop SR, Pivik J (1995) The Pain Catastrohizing Scale: development and validation. Psychol Assess 7:524-532

26. Mascagni T, Bijttebier P, Crombez G, Vlaeyen J (2001) De pain catastrophizing scale for children: eerste psychometrische bevindingen. Gedragsther 34:325-336

27. Verstraeten K, Bijttebier P, Crombez G, Mertens G, Mascagni T, Goubert L (2003) De pain catastrophizing scale for children: predictieve validiteit in een klinische steekproef. Gedragsther 36:299-307

28. Carver CS, White TL (1994) Behavioral inhibition, behavioral activation, and affective responses to impending reward and punishment. J Pers Soc Psychol 67:319-333

29. Muris P, Meesters C, De Kanter E, Eek Timmerman P (2005) Behavioural inhibition and behavioural activation system scales for children: relationships with Eysenck's personality traits and psychopathological symptoms. Pers Individ Diff 38:831-841

30. Capaldi DM, Rothbart MK (1992) Development and validation of an early adolescent temperament measure. J Early Adolesc 12:153-173

31. Ellis LK, Rothbart MK (2001) Revision of the early adolescent temperament questionnaire. Poster presented at the Biennial Meeting of the Society for Research in Child Development. Minneapolis, Minnesota

32. Goodman R (2001) Psychometric properties of the strengths and difficulties questionnaire. J Am Acad Child Adolesc Psychiatry 40:1337-1345

33. Campo JV, Fritsch SL (1994) Somatization in children and adolescents. J Am Acad Child Adolsc Psychiatry 33:1223-1235

34. Palermo TM (2000) Impact of recurrent and chronic pain on child and family daily functioning: a critical review of the literature. J Dev Behav Pediatr 21:58-69

35. Nitch SR, Boone KB (2004) Normal personality correlates of chronic pain subgroups. J Clin Psychol Med Sett 11:203-209

36. Tallis F, Eysenck MW (1994) Worry: mechanisms and modulating influences. Behav Cogn Psychother 22:37-56

37. Hamilton NA, Zautra AJ, Reich JW (2005) Affect and pain in rheumatoid arthritis: do individual differences in affective regulation and affective intensity predict emotional recovery from pain? Ann Behav Med 29:216-224

38. Muris P, Van Zuuren FJ (1992) Monitoring, medical fears, and physical symptoms. Br J Clin Psychol $31: 360-362$ 
39. Crombez G, Eccleston C, Van den Broeck A, Goubert L, Van Houdenhove B (2004) Hypervigilance to pain in fibromyalgia: the mediating role of pain intensity and catastrophic thinking about pain. Clin $\mathrm{J}$ Pain 20:98-102

40. McDermid AJ, Rollman GB, McCain G (1996) Generalized hypervigilance in fibromyalgia: evidence for perceptual amplification. Pain 66:133-144

41. Keefe F, Rumble M, Scipio C, Giordano L, Perri L (2004) Psychological aspects of persistent pain: current state of the science. J Pain 5:195-211

42. Eccleston C, Morley S, Williams A, Yorke L, Mastroyannopoulou K (2002) Systematic review of randomnized controlled trials of psychological therapy for chronic pain in children and adolescents, with a subset meta-analysis of pain relief. Pain 99:157-165 